\title{
Functional Organization of Human Motor Cortex: Directional Selectivity for Movement
}

\author{
Michal Eisenberg, ${ }^{1,2 \star}$ Lior Shmuelof, ${ }^{1 \star}$ Eilon Vaadia, ${ }^{2,3}$ and Ehud Zohary ${ }^{1,2}$ \\ ${ }^{1}$ Department of Neurobiology, Life Sciences Institute and 2 Interdisciplinary Center for Neural Computation, Hebrew University, Jerusalem 91904, Israel, \\ and ${ }^{3}$ Department of Physiology, Hadassah Medical School, Hebrew University, Jerusalem 91120, Israel
}

In monkeys, neurons in the hand representation of the primary motor cortex (M1) are often tuned to the direction of hand movement, and there is evidence that these neurons are clustered according to their "preferred" direction of movement. However, this organizational principle has yet to be demonstrated in M1 of humans. We conducted a functional magnetic resonance imaging (fMRI) study in which participants used a joystick to move a cursor from a central origin to one of five equidistant targets. The fMRI signal of individual voxels was sensitive to the directional aspects of the reaching task and manifested direction-specific adaptation. Furthermore, the correlation between multivoxel patterns of responses for different movement directions depended on the angular distance between them. We conclude that M1 neurons are likely to be organized in clusters according to their preferred direction, since only such a coarse-grained representation can lead to directional selectivity of voxels, encompassing millions of neurons. A simple model that estimates cluster size suggests that the diameter of these clusters is on the order of a few hundred micrometers.

\section{Introduction}

Neurons in the primary motor cortex (M1) of monkeys are tuned to the direction of limb movement (Georgopoulos et al., 1982). Single-cell recordings from M1 suggest that these neurons are organized according to their preferred directions (PDs) (Asanuma and Rosén, 1972; Amirikian and Georgopoulos, 2003; Ben-Shaul et al., 2003; Georgopoulos et al., 2007). Directional tuning has also been found in the multiunit activity recorded from M1 (Stark et al., 2009), as well as from local field potentials (LFPs) (Mehring et al., 2003; Rickert et al., 2005), which represent populations at a resolution of $\sim 1 \mathrm{~mm}$ (Berens et al., 2008; Rasch et al., 2009). Together these data suggest that in monkeys, M1 neurons are clustered, to some extent, according to their PDs. This organizational feature resembles the columnar organization characteristic of somatosensory cortex (Powell and Mountcastle, 1959), primary visual cortex (V1) (Hubel and Wiesel, 1962), middle temporal cortex (MT) (Albright et al., 1984), and primary auditory cortex (Imig and Adrian, 1977).

Obviously, little is known about the properties of neurons in the homologous area (M1) of humans. A recent study, performed for clinical purposes, showed that human M1 neurons are also often directionally tuned (Truccolo et al., 2008). It is less clear,

Received Jan. 3, 2010; revised March 15, 2010; accepted March 26, 2010.

This research was supported by an Israel Science Foundation Grant 39/09 to E.Z. We thank I. Nelken, S. Wise, Y Pertzov, E. Stark, H. Sompolinski, and D. Shore for their insightful comments and Eldad Assa and Yuval Link for programming the task.

*M.E. and L.S. contributed equally to this work.

Correspondence should be addressed to Michal Eisenberg, Department of Neurobiology, Life Sciences Institute and Interdisciplinary Center for Neural Computation, Hebrew University, Jerusalem 91904, Israel. E-mail: michal. eisenberg@mail.huji.ac.il.

L. Shmuelof's present address: Motor Performance Lab, Neurology Department, Columbia University, New York, NY 10032.

D0I:10.1523/JNEUROSCI.0007-10.2010

Copyright $\odot 2010$ the authors $\quad 0270-6474 / 10 / 308897-09 \$ 15.00 / 0$ however, whether the neurons are organized in functionally related clusters, such that neighboring neurons share similar tuning properties. To address these issues, we used functional imaging techniques coupled with multivoxel pattern analysis. This approach seems, at first, unlikely to reveal functional clustering in $\mathrm{M} 1$, if only because the spatial resolution of functional magnetic resonance imaging (fMRI) (several millimeters) is much larger than the size of functional units in the cortex. For example, the typical diameter of a cortical column in primary visual cortex, defined on the basis of orientation selectivity, measures hundreds of micrometers in diameter (Berman et al., 1987). Nevertheless, recent applications of multivoxel pattern analysis in imaging studies allowed the detection of columnar organization in visual areas such as V1 (Kamitani and Tong, 2005) and MT (Kamitani and Tong, 2006). In this study, we use these analysis methods to test the hypothesis that neurons cluster according to their directional preferences in the human M1 cortex. We reasoned that examination of directional preferences at the voxel level could support this hypothesis, provided that the number of clusters within a voxel is small. In this case, random fluctuations in the number of clusters with preference for a specific direction, together with the natural tuning characteristics of the neurons, might determine the directional preference of the voxel as a whole.

To that end, our participants performed a "center-out task," similar to the one performed by monkeys in studies of M1 (Georgopoulos et al., 1982). During an event-related fMRI scan, our participants repeatedly moved a cursor from the center of a screen toward various targets in the periphery by moving a joystick in the corresponding direction. We found (1) that M1 voxels were selective for the directional aspects of the reaching task, (2) that the patterns of activation across M1 voxels became less correlated as the angular difference between movements in- 
creased, and (3) that direction-specific fMRI adaptation [i.e., repetition suppression (RS)] occurred when the same movement was repeated in the following trial. These results support the hypothesis that human M1 is organized in clusters of neuronal populations with similar PDs of movement.

\section{Materials and Methods}

Participants. Eleven right-handed volunteers with normal or corrected-to-normal visual acuity and no neurological or psychiatric history ( 5 women and 6 men, aged 18-35) participated in the present experiments. Hadassah Ein Kerem Medical Center Ethics Committee approved the experimental procedure. Written informed consent was obtained from each participant.

MRI acquisition. The blood oxygenation level-dependent (BOLD) fMRI measurements were performed in a whole-body $3 \mathrm{~T}$ Trio Siemens scanner. The functional MRI protocols were based on a multislice gradient echoplanar imaging and a standard head coil. The functional data were obtained under the optimal timing parameters: $\mathrm{TR}=2000 \mathrm{~ms}, \mathrm{TE}=$ $30 \mathrm{~ms}$, flip angle $=90^{\circ}$, imaging matrix $=80 \times$ 80 , voxel size: $2.75 \times 2.75 \times 3.1 \mathrm{~mm}$. The 30 slices (with a gap of $0.3 \mathrm{~mm}$ ) were oriented in the axial direction. The scan covered the whole brain. Each participant was scanned in one run lasting $20.5 \mathrm{~min}$. The run was comprised of an acquisition of 616 volumes and contained 250 trials.

Experimental paradigm. Each trial lasted $4 \mathrm{~s}$. The trial began with presentation of a red circle in the center of the screen ("origin," radius of $0.7^{\circ}$ ). Initially, the participants had to hold an MRI-compatible joystick still, and make no hand movement. After an interval of $500 \mathrm{~ms}$, five circles (targets, radius of $1^{\circ}$ ) appeared at the upper half of the screen, spread around the center at equal distances, between $0^{\circ}$ and $180^{\circ}, 45^{\circ}$ apart. Their distance from the origin was $4.5^{\circ}$ of visual angle (Fig. 1). Four of the circles were blue and one circle was green, signaling the required future direction of movement. The participants had to keep their hand still until the "go" signal, in which the red "origin" circle turned into a white cursor, which occurred $2 \mathrm{~s}$ later. They were instructed to respond by moving the cursor toward the green target, using the joystick (Fig. 1a). Participants had $1.5 \mathrm{~s}$ to reach the target. Upon reaching the target, all circles disappeared. This served as a cue for the participants to release the joystick (thereby relaxing the spring), which resulted in the joystick returning to its starting position at the center. There was no explicit failure signal. If the participants did not reach the target within the $1.5 \mathrm{~s}$ time limit, all circles disappeared and the next trial began (participants failed to reach the target in $10.6 \%$ of the trials). There were also intermittent null trials in which the red origin and all five blue targets appeared on the screen for 2-8 s (thus no target was distinctly marked for movement). These trials were obviously not followed by a "go" signal. During these null trials, the participants were instructed not to move the joystick until the next trial began. The task was programmed with MATLAB version 7.1 (MathWorks), using Psychtoolbox (Brainard, 1997; Pelli, 1997).

This rapid event-related fMRI study consisted of 250 trials, 50 in each direction. The trial order was counterbalanced (first order) and embedded with null trials of various lengths $(2,4,6$, or $8 \mathrm{~s})$. The different lengths of the null trials were used to randomize the timing of the movement trials, which allow a more efficient estimation of activation (Dale, 1999). Null trials were pseudorandomly embedded between movement trials. Our constraint was that the total length of all null trials would equal the total length of each of the five movement conditions (200 s). The trial sequences were built using optseq software, which chooses the most efficient sequence (most variable history before each condition) out of
10,000 random sequences sampled. The experiment began and ended with $16 \mathrm{~s}$ of a null event.

We used only five directions of movement, covering only half of the plane, instead of eight targets covering the entire plane, to achieve as many trials as possible for each condition to obtain a reliable signal. We also chose to use targets with rather small angular differences $\left(45^{\circ}\right)$ rather than spanning the entire plane with larger differences $\left(72^{\circ}\right)$. This was done to allow us to assess not only the selectivity for a specific direction of movement, but also the relationship between voxel representations for similar movements (to neighboring targets).

Data analysis. Preprocessing and defining regions of interest (ROIs) was done using Brain Voyager QX (Brain Innovation).

The functional images were superimposed on two-dimensional anatomical images and incorporated into the three-dimensional datasets through trilinear interpolation. Before statistical analysis, head motion correction and high-pass temporal filtering in the frequency domain (3 cycles/total scan time) were applied to remove drifts and to improve the signal-to-noise ratio. The complete dataset was transformed into three-dimensional Talairach space with a resolution of $3 \times 3 \times 3 \mathrm{~mm}^{3}$.

The left M1 ROI was individually defined for each participant as a cluster within the central sulcus, which showed higher activation during movement than during rest (general linear model, $p<10^{-8}$; Bonferroni corrected, $p<0.01)$. The average size of M1 was $140 \pm 40$ functional voxels (see supplemental Table 1, available at www.jneurosci.org as supplemental material).

Further analysis was done using Matlab R2007b (MathWorks). We used linear regression to estimate response amplitudes ( $\beta$ values) for each functional voxel in each condition, solving an equation of the form $\boldsymbol{y}=\mathbf{X} \boldsymbol{b}+\boldsymbol{e}$, where the vector $\boldsymbol{y}$ is the measured voxel time course, the vector $\boldsymbol{b}$ contains a sequence of the estimated response amplitudes for each of the five conditions, $\mathbf{X}$ is the convolution matrix determined by the sequence of events, and $\boldsymbol{e}$ is the error (Gaussian noise). The convolution matrix, $\mathbf{X}$, was designed with predictors of onset times for each trial and has the dimensions of $614 \times 51$. $\mathrm{X}$ contains a row for each time point (TR) in the experiment (totaling 614) and 10 columns for each of the 5 conditions (or conditions in the correlation analyses) plus a column of 
ones for the offset predictor (thus 51 columns). This leads to an independent estimation of the response amplitude, for each condition at each time point (the first point out of the 10 points for each condition is the time of the beginning of a trial). The vector of response amplitudes, $\boldsymbol{b}$, is estimated using the equation $\boldsymbol{b}=\left(\mathbf{X}^{\prime} \mathbf{X}\right)^{-1} \mathbf{X}^{\prime} \boldsymbol{y}$ and is respectively comprised of 10 values (estimated $\beta$ values) for each condition (one for each time point between 0 and $18 \mathrm{~s}$ after the beginning of the trial). The first value of $\boldsymbol{b}$ is the mean activation over the entire time course. Importantly, since we estimated the BOLD activation for each time point separately, no assumptions were made about the shape of the hemodynamic response. Linear regression was significant in $>99 \%$ of voxels. On average, the variance explained by the model accounted for $37 \%$ of the total variance.

Coefficient of variation analysis. We used a bootstrap analysis, to test whether the coefficient of variation $(\mathrm{CV})(\mathrm{SD} /$ mean) of the five directions of movement in each voxel is significantly higher than expected by mere chance (e.g., had there been no directional selectivity). In this analysis, we randomly reassigned conditions (directions of movement) to the trials, while maintaining the original proportions of hand movements (each condition was assigned to $20 \%$ of the trials). The null conditions were not replaced. We created a new regression matrix, estimated the $\beta$ values for the different directions, and assessed the $\mathrm{CV}$, separately for each assignment. This procedure was repeated 10,000 times, resulting in a distribution of expected CVs merely due to noise. Next, for each voxel, we assessed its $p$ value: the fraction of $\mathrm{CV}$ values obtained by the bootstrap method that were greater than the actual CV of that voxel. Finally, a $\chi^{2}$ test was used to show that the distribution of $p$ values across voxels (shown in supplemental Fig. 3, available at www.jneurosci.org as supplemental material) was significantly skewed to low values (differing from a uniform distribution, which would be expected by chance).

The CV analysis was used also for the estimation of cluster size (see Discussion and Fig. 7). For this purpose, since each voxel is expected to show some variation in its response by chance (as there were limited repetitions of each direction of movement), we also corrected each voxel's $\mathrm{CV}$ by subtracting from its actual variance in response (for the various directions) the mean variance of the bootstrapped activation $\left(X_{\mathrm{BS}}\right)$ across iterations:

$$
\mathrm{CV}=\frac{\sqrt{\left(\operatorname{Var} \boldsymbol{B}-\operatorname{mean}\left(\operatorname{Var} \boldsymbol{B}_{\mathrm{BS}}\right)\right)}}{\operatorname{mean}(\boldsymbol{B})} .
$$

In addition to calculating voxel $\mathrm{CV}$, we also calculated the $\mathrm{CV}$ as a measure of the directionality of the LFPs in monkey M1, when monkeys performed the same center-out task [courtesy of S. Cardoso de Oliveira (Cardoso de Oliveira et al., 2001)]. To that end, we used the peak-to-peak distance (the distance between maximum and minimum) of the mean evoked potential, per direction of movement. CVs of LFPs were calculated without subtracting bootstrapped variance. Consequently, the CV is slightly overestimated.

Analysis of spatial patterns of $\mathrm{fMRI}$ response. To detect directional selectivity of voxel population spatial patterns, each individual's data were split into two datasets, such that each of the 50 trials in each direction was randomly assigned to one of the datasets. For each voxel, we estimated the $\beta$ values and defined the activation for each direction (i.e., activation value) as the average of the $\beta$ values measured 6 and $8 \mathrm{~s}$ after the beginning of the trial $(\sim 4-6 \mathrm{~s}$ after movement initiation; normally at the peak activation) (see Fig. $2 b$ ). Then, in each dataset and for each voxel, we subtracted the voxel's mean activation level (across all directions) to remove activation differences between voxels that are unrelated to movement direction. Without such a correction procedure, one would get high correlations between the multivoxel spatial patterns from all comparisons, simply because some voxels are more active than others, regardless of the direction of movement.

The above analysis resulted in two matrices, one for each dataset. Each matrix consisted of 5 columns (one for each direction) with length $N$ equal to the number of voxels in the ROI. The entries of each row of the matrix were the 5 activation values of a single voxel for all 5 directions of movement.

Next, we calculated the correlation coefficient (CC) between the columns of the first and the second datasets (each corresponding to the pattern of activation across all voxels, for a given condition). If the activation values contain information about the direction of movement, one should get a higher CC for movements to the same direction (in the two datasets) than the CC calculated for movements to two different directions. To address the concern of low-frequency temporal trends in our data, we repeated this analysis 100 times for each participant; in each iteration, the data were split differently into two random datasets. The results shown are the mean CC values across all 11 participants. The resulting dependence of the $\mathrm{CC}$ on the angular difference between the two directions of movements matched a normal distribution:

$$
y_{\mathrm{i}}=h \times e^{-\frac{\left(x_{\mathrm{i}}-\mu\right)^{2}}{\sigma^{2}}}-b .
$$

Analysis of spatial patterns of simulated data. Individual maps often display a preference for some directions over others. In other words, the distribution of preferred directions across voxels was not uniform (though the identity of the most common preferred direction varied among participants). This naturally raises the concern that the information about movement direction could potentially be due to artifacts at a coarse scale (such as large blood vessels, which may affect the activation of many voxels). To address this concern, we performed a new analysis in which we used simulated data. Simulation of the data was done by replacing the activation values for each direction (across voxels) with random values taken from a normal distribution with the same mean and SD as the real data for that direction. Now tuning of voxels is due only to the global bias of one direction over others in the entire ROI. We ran the multivoxel pattern analysis with the simulated data. Tuning of the multivoxel pattern would imply that our results do not stem from any organization at the subvoxel level, but rather from a large-scale variability between directions (Op de Beeck, 2010). See supplemental Figure 2 (available at www.jneurosci.org as supplemental material) for results.

Movement repetition analysis. In the repetition analysis, the various conditions were also split according to the angular difference between the direction of movement in the current trial and the movement direction in the previous trial. Thus, a condition of $0^{\circ}$ means that the movement was to the same direction as in the previous trial. Beta values were estimated in the same way as in the previous analyses.

To test whether a voxel's PD (direction eliciting the greatest response) is the same direction that elicits the greatest repetition suppression (RS), we divided the trials into 10 conditions. First we divided the trials into repeated and nonrepeated trials, and then we divided each set of trials into five conditions according to the direction of movement. The repetition index for each voxel $(v)$ and each direction $(d)$ was defined as $\beta_{v, d}$ (nonrepeated) $/ \beta_{v, d}$ (repeated).

Both repetition index and activation index (of nonrepeated trials) were normalized by subtracting the mean and dividing by the SD across directions in each voxel to bring the two different signals to a common scale and then correlated. Pairwise Student's $t$ test was used to test whether the CCs in the "same direction" and "different direction" conditions were significantly different.

\section{Results}

Participants performed a "center-out" task in a rapid eventrelated fMRI experiment. In this task, they had to move a cursor from the center of a video screen toward one of five equidistant targets located at the periphery of the screen. On each trial, one of the five targets had a different color than the rest, to indicate that it was to be the future target, and the participants later used an MRI-compatible joystick to move the cursor to that location on the screen (Fig. 1a). They made a series of center-out movement trials in the five different directions while being scanned. Null trials (with no movement) were also embedded in the scan pseudorandomly. Each movement was performed 50 times in a counterbalanced order during the course of a $20.5 \mathrm{~min}$ scan. The participants reached the target correctly on $89.4 \%$ of the trials. The angular deviation of movement was assessed for each trial by calculating the angle between the actual trajectory at the peak 

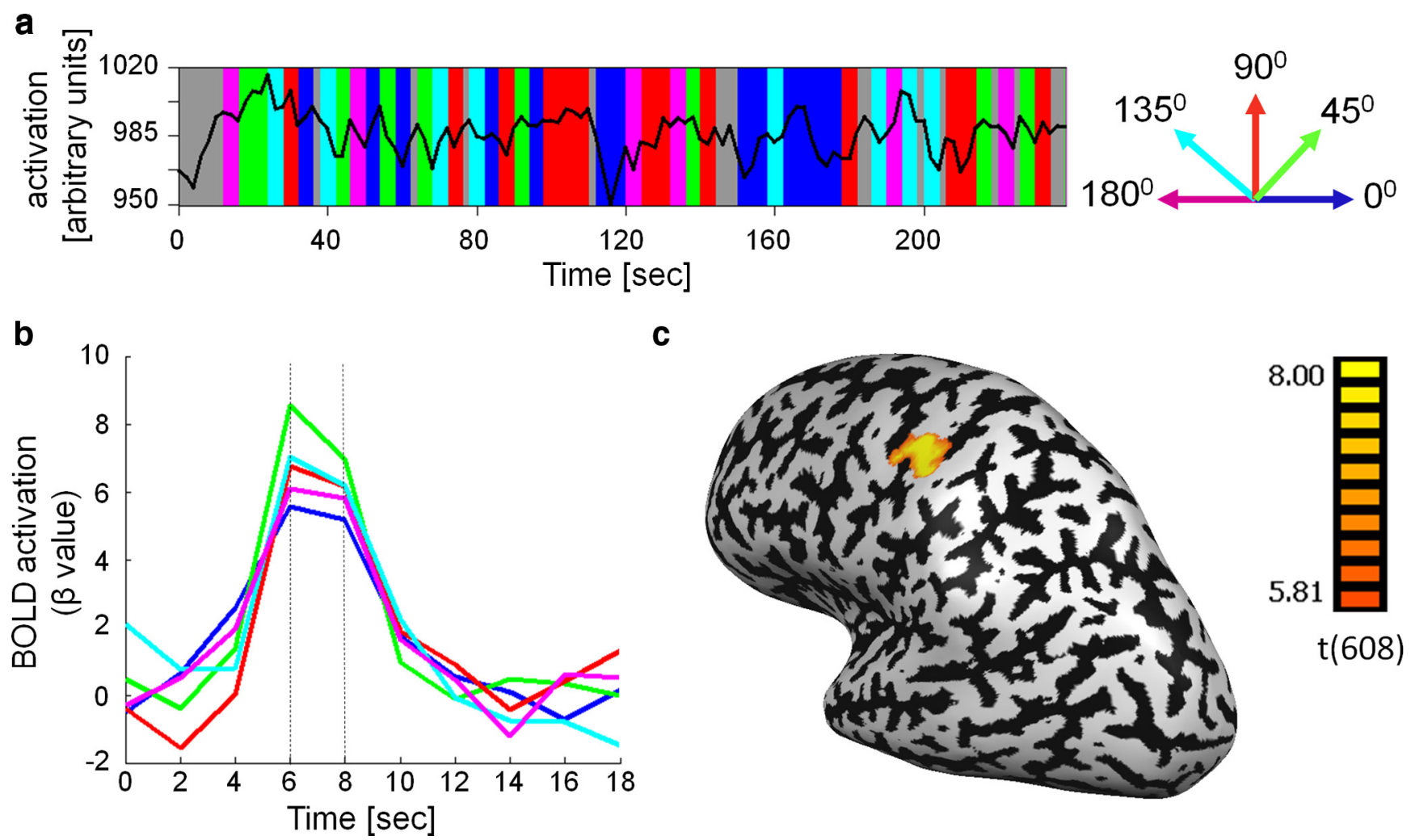

C
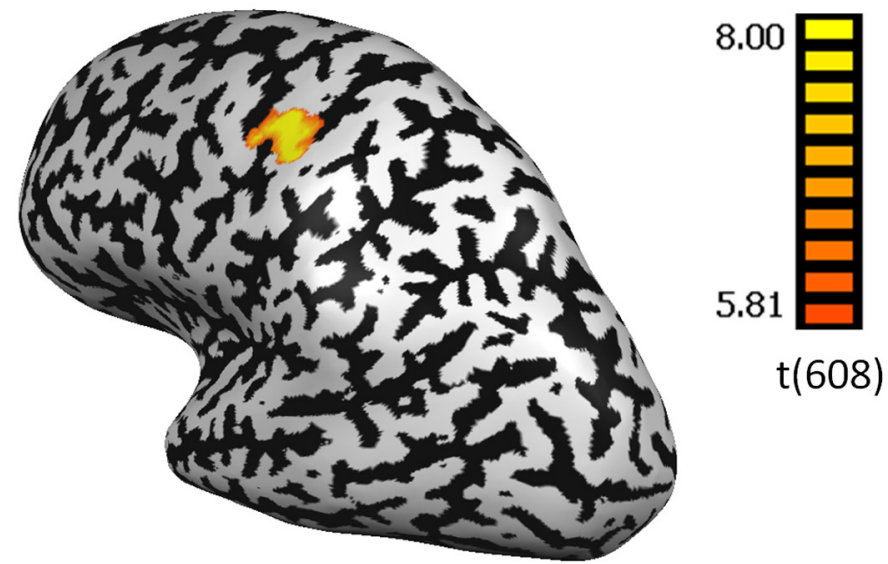

Figure 2. Extraction of the hemodynamic response kernels for the different movement directions. $\boldsymbol{a}$, The time course of activation of an example voxel. Each color denotes movement in one of the five directions according to the arrows on the right. The direction of movement was randomized across trials, interspersed with null (no-movement) trials (in gray). In this example only the first $240 \mathrm{~s}$ of the session are shown. $\boldsymbol{b}$, The extracted hemodynamic response kernel for each movement direction (using the deconvolution method). Colors correspond to the movement directions depicted in $\boldsymbol{a}$. For each direction, the mean activation level (estimated $\beta$ value) between the two dashed lines was used as the voxel's activation value for further analysis. $\boldsymbol{c}$, Definition of left M1. ROls were defined for each participant individually as a cluster of voxels within the central sulcus of the left hemisphere, which showed higher activation during movement than during rest $(p<$ $10^{-8} ;$ Bonferroni corrected, $\left.p<0.01\right)$.

velocity and the direct trajectory to the center of the target. The mean absolute angular deviation was $10.4^{\circ}$ (Fig. $1 b$ ).

We defined cortical regions involved in the visuomotor reaching task by identifying voxel clusters that exhibited significant enhanced activity during the task compared with null trials $(p<$ $10^{-8}$; Bonferroni corrected at least $p<0.01$ ). Several ROIs were defined for each participant, but here we focus on the results from left M1 (Fig. 2c).

The resulting time course of activation for each voxel, acquired during the scan, was further analyzed using linear regression to estimate the profile of activation generated by each of the five directions of movements. An example of a voxel's time course of activation and the extracted convolution kernels (hemodynamic response) to the different directions are shown in Figure 2. For each voxel, the average of the estimated $\beta$ values measured 6 and $8 \mathrm{~s}$ after the beginning of the trial (which were usually the time points of peak activation) served as the extracted parameter of activation level, for each direction.

\section{Voxel tuning analysis}

We began by inspecting the degree of directional tuning in each voxel. Note that reaching movements were made only to targets in the upper half of the screen (corresponding to forward joystick movements). Therefore, we could not use the classical estimation of tuning, i.e., cosine fits (Georgopoulos et al., 1982), and had to use other measures instead. For each voxel, we computed the CV, which is the SD of the voxel's activation level (across different directions), divided by its mean activation level (across those directions). Next, we assessed the probability that each of the voxels would exhibit directional specificity simply by chance, by conducting a bootstrap test in which the direction of each movement was randomly reassigned (in the regression matrix). This analysis revealed that $28 \%$ of the voxels in M1 were directionally selective (i.e., they had a CV greater than $95 \%$ of the CV values obtained by the bootstrap method). A $\chi^{2}$ test showed that the distribution of $p$ values across voxels (supplemental Fig. 3, available at www.jneurosci.org as supplemental material) was significantly skewed to lower values from those expected by chance $\left(\chi_{(9)}^{2}=1386 ; n=1542 ; p<0.001\right)$. Note also that since not all directions of movement were tested, this percentage is likely to be a lower bound on the percentage of voxels sensitive to movement direction.

Next, we aligned all the voxels' activation tuning curves according to their PD to obtain an average voxel tuning curve (Fig. 3 ). This computation allowed us to assess whether the average voxel's preference is only for a single, specific $\mathrm{PD}$ or whether there is instead gradual tuning at the voxel level. In the latter case, movement in directions close to the PD, in angular terms, should elicit a greater BOLD response than movements far from the PD. Figure $3 b$ shows that the BOLD signal decreases with angular distance from the voxel's PD. A one-way repeated-measures ANOVA with direction difference as the relevant factor, taking into account only the BOLD signal for movements with an angular difference of $45^{\circ}, 90^{\circ}$, and $135^{\circ}$ apart, indicated that this factor was marginally significant $\left(F_{(2,20)}=3.29, p<0.058\right)$ (movements with a $0^{\circ}$ difference were discarded from this analysis be- 
a

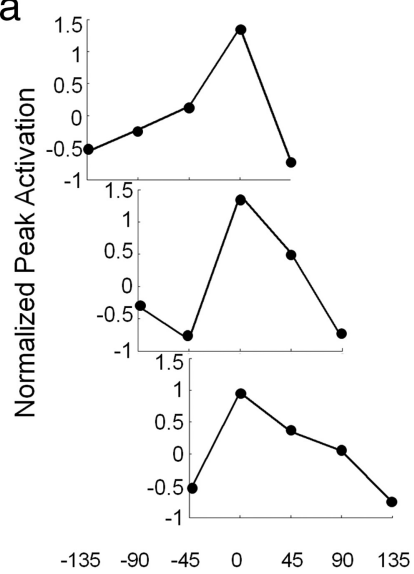

Angular distance from PD [degrees] b

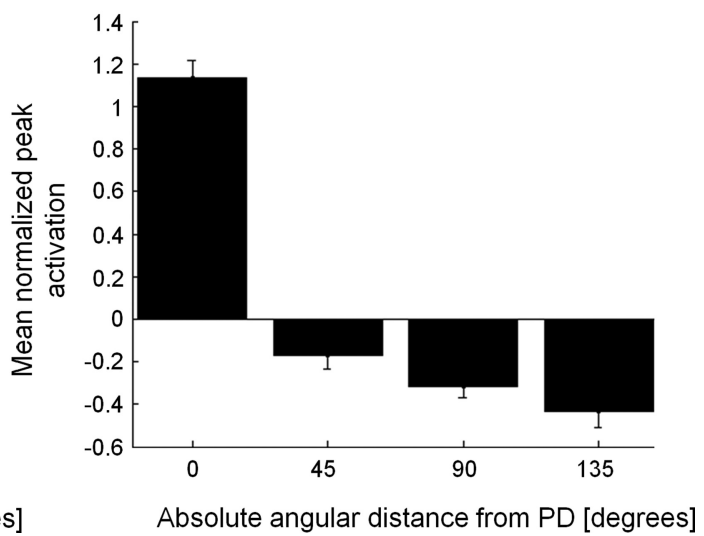

Figure 3. M1 voxels show directional tuning. $\boldsymbol{a}$, Example of individual voxel "tuning curves" showing the BOLD activation of 3 voxels as a function of movement direction. Each tuning curve was normalized such that the mean activation of each voxel across all directions was zero. In each participant, the "tuning curves" of all individual voxels from M1 were aligned according to their PD, to generate a "voxel population tuning curve." This population tuning curve was averaged across participants. $\boldsymbol{b}$, The resulting "average voxel" activation as a function of the angular distance from the PD. The peak at the PD is a direct result of the alignment procedure, but note the smooth tuning preference as the angular distance between directions of movement increases. Error bars denote SEM across participants.

a

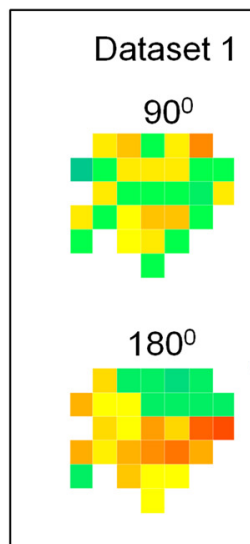

b

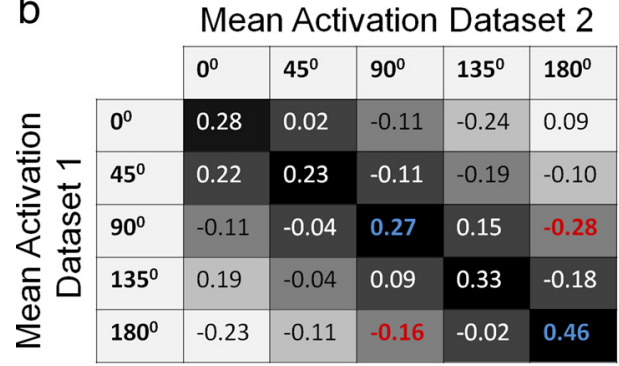

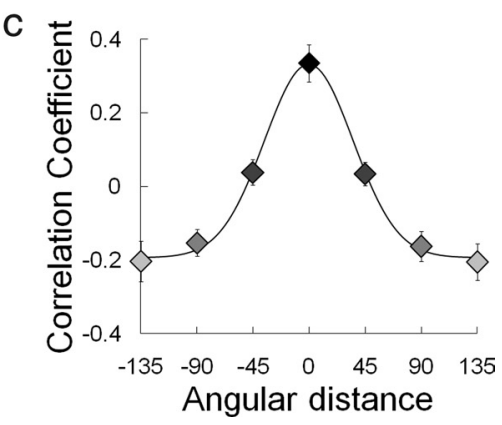

Figure 4. Multivoxel spatial patterns of activation are more similar for closer movements. $\boldsymbol{a}$, Example of the patterns of $M 1$ activation in one subject for movements in $90^{\circ}$ (top) and $180^{\circ}$ (bottom), in the two datasets. These examples (shown mainly for illustrative purposes) are taken from the axial plane (Talairach coordinates: $z=50$ ) from one of the participants. Blue arrows denote correlations between movements in the same directions ( 0.33 and 0.56 for this participant), whereas red arrows denote correlations between movement in the different directions $(-0.43$ and -0.31$)$. $\boldsymbol{b}$, Matrix of the average CCs between the multivoxel patterns in the two datasets (across all participants), for all possible direction combinations. The main diagonal corresponds to CCs between same directions; the next diagonals (in gray) correspond to the CC between directions $45^{\circ}$ apart, etc (in lighter colors). Red and blue values correspond to the average value (across participants) for the directions in the example (a). $\boldsymbol{c}$, The similarity between patterns of voxel activations (measured by the $\mathrm{CC}$ ) decreases as a function of the angular distance between the two directions of movements. The grayscale of each data point (diamonds) indicates the appropriate diagonal in $\boldsymbol{b}$ whose average value is depicted. Error bars denote SEM across participants. The black curve denotes the Gaussian fit to the CCs. cause, due to our alignment procedure, their BOLD signal is necessarily greater than the other angular distances; movements with a difference of $180^{\circ}$ from PD were discarded as well because there were considerably fewer cases than other directions).

\section{Multivoxel pattern analysis}

A different approach to study the tuning properties of voxels in a given region is to calculate the correlation between the multivoxel spatial patterns of activation for the different directions. Having shown that voxels show some directional tuning, one may expect to find greater correlation between multivoxel spatial patterns of activations during movements with a smaller angular difference.

To that end, two non-overlapping datasets were created by randomly assigning each of the 50 trials in each direction into one of the two categories. Next, we used linear regression to estimate the level of activation of each voxel in each movement direction separately for each dataset. Finally, to eliminate differences in activation between voxels, in each dataset we subtracted the mean response of each voxel (across the various directions). This resulted in two vectors of activation (across voxels) for each direction of movement, corresponding to the two datasets (see PD maps in supplemental Fig. 1, available at www.jneurosci.org as supplemental material). We then calculated the correlation coefficient $(r)$ between the activation vectors for each pair of directions from the two sets. (For example, we calculated the correlation between activation elicited by movements toward the $90^{\circ}$ target in the first set and movements toward the $180^{\circ}$ target in the second set, or by movements elicited in the same direction; see examples in Fig. 4a.)

This resulted in a matrix of correlation coefficients for all possible comparisons (Fig. 4b). Next, we averaged across conditions having the same angular difference (diagonals of the matrix in Fig. $4 b$ ) to obtain the mean correlation (between patterns) as a function of the angular distance between the trajectories: the average correlation tuning curve (Fig. $4 c$ ). As the distance between the directions of movement increased, the correlation decreased (Gaussian fit, $r=0.999$ ).

It is possible that the directional selectivity at the level of representation of voxel populations could simply be a side effect of an uneven distribution of PDs across voxels. To test this, we ran the correlation 
analysis using simulated data that preserved the original uneven distribution across voxels. We found no tuning of the correlation coefficients of the patterns of activation for the simulated data (supplemental Fig. 2, available at www.jneurosci. org as supplemental material). We conclude that our results reflect information based on the direction tuning at the voxel level, rather than a global bias in preference toward some directions over others.

\section{Effect of movement RS}

Another way to study the selectivity of BOLD activation to the direction of movement is to examine the effect of movement repetition. RS, the reduction in the BOLD signal following repeated presentation of the same stimulus, was initially reported in visual cortex (Grill-Spector and Malach, 2001). It was recently documented for motor actions as well (Dinstein et al., 2008). One might therefore expect to find greater reduction of the BOLD signal in M1 when the same movement is repeated across trials. To test this, we categorized the trials according to their similarity to the previous trial. Figure 5 shows the average time course of activation across M1 voxels (Fig. $5 a$ ) and the mean BOLD activation (Fig. 5b) as a function of the angular difference from the previous trial. The BOLD activation is lower when the participant made a movement toward the same target as in the previous trial (one-way repeated-measures ANOVA, $F_{(4,40)}=5.58$, $p<0.005$, Tukey-Kramer post hoc test, $p<0.005)$. This phenomenon seems to be limited to the same direction, and does not generalize to near targets (i.e., there is no statistical difference in the BOLD activation for the current movement when the previous movement diverged from it by $45^{\circ}, 90^{\circ}$, or $\left.135^{\circ}, F_{(3,30)}=0.4, p=0.75\right)$.

Is the preferred direction of the voxel (eliciting the greatest response) also the direction that elicits the greatest RS? If so, repeated movements toward the voxel's PD (direction with maximum activation) should lead to greater RS than repeated movements to other directions. To that end, we assessed the correlation between the pattern of multivoxel activation for a given direction and the pattern of multivoxel RS for that direction, as well as for all other directions (see example for the $45^{\circ}$ and $90^{\circ}$ movement cases in Fig. $6 a$ ). This resulted in a matrix of correlation coefficients between the repetition suppression and activation level for all possible movement pairs. Our findings show that the level of activation and RS are positively correlated for same directions (mean $\mathrm{CC}=0.29, \mathrm{SD}=0.09$ ), and significantly greater than different directions (mean $\mathrm{CC}=$ $\left.-0.07, \mathrm{SD}=0.02 ; t_{(10)}=10.12, p<5 \times 10^{-6}\right)$. To verify that our results did not stem merely from use of the same data for

a

b between same directions.
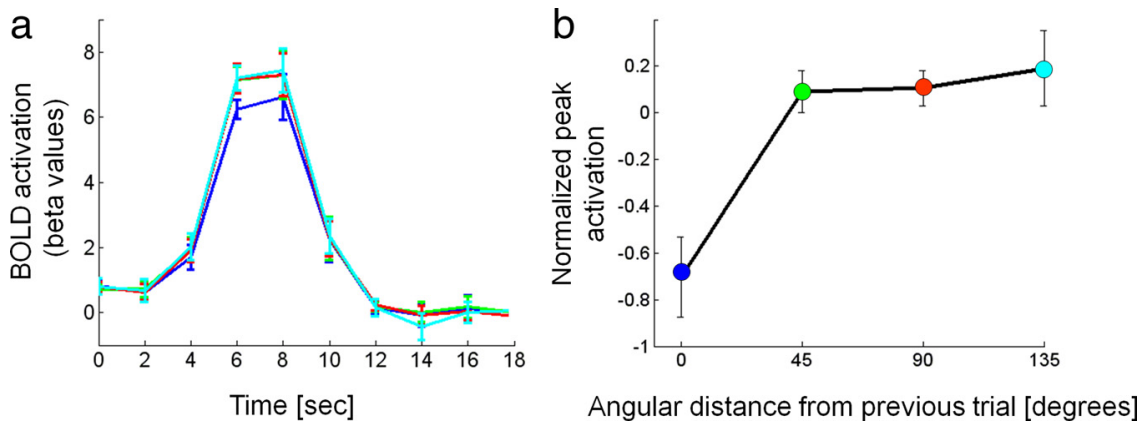

Figure 5. RS of the $\mathrm{fMRI}$ response in primary motor cortex. $\boldsymbol{a}$, The various colors denote the mean time course of activation in the M1 R0I (across participants) for a movement in a given direction when preceded by a movement in the same direction in the previous trial (blue line) or a different direction (green, red, and cyan denote cases in which the previous trial was $45^{\circ}, 90^{\circ}$, and $135^{\circ}$ apart from the current trial, respectively). Suppression is seen only for cases of repetition of the same movement. $\boldsymbol{b}$, The mean level of activation (measured at time points 6 and $8 \mathrm{~s}$ and then normalized such that the mean of each voxel across directions was 0 ) as a function of the absolute angular difference between the current movement and the movement in the previous trial. Colors correspond to the conditions shown in $\boldsymbol{a}$. Error bars denote SEM between participants.

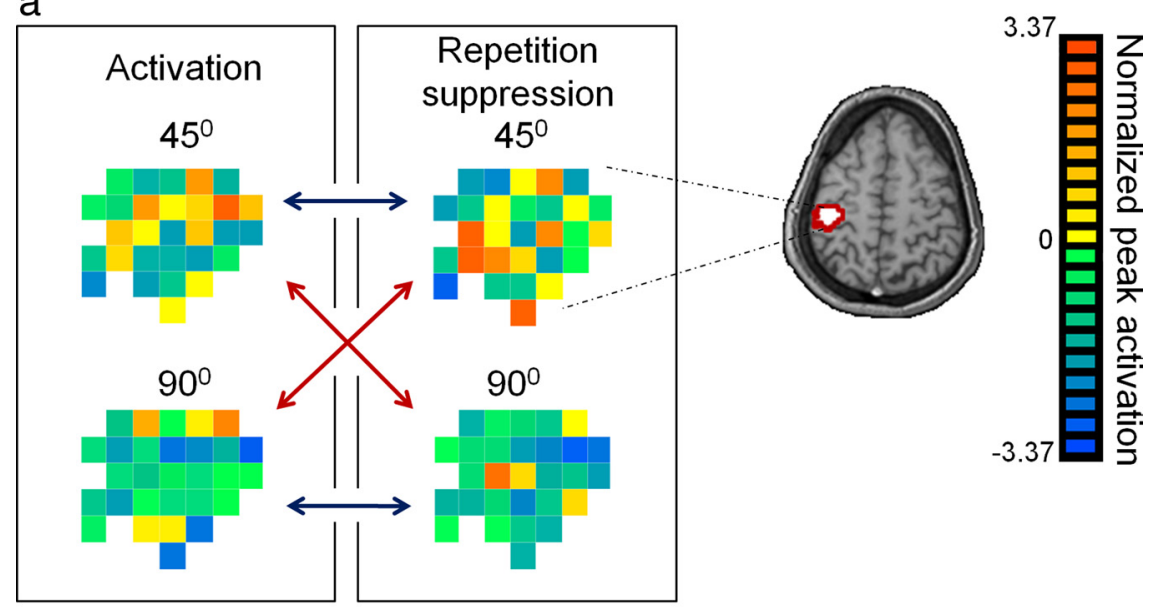

\begin{tabular}{|c|c|c|c|c|c|c|}
\hline \multirow{2}{*}{\multicolumn{2}{|c|}{$ᄃ \quad 0^{0}$}} & $0^{0}$ & $45^{0}$ & $90^{\circ}$ & $135^{\circ}$ & $180^{\circ}$ \\
\hline & & 0.34 & -0.28 & -0.02 & 0.01 & -0.02 \\
\hline \multirow{2}{*}{ 䓠 } & $45^{0}$ & -0.18 & 0.42 & -0.08 & -0.09 & -0.04 \\
\hline & $90^{\circ}$ & -0.18 & -0.04 & 0.33 & -0.16 & 0.02 \\
\hline \multirow{2}{*}{ 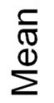 } & $135^{\circ}$ & 0.08 & -0.09 & -0.16 & 0.20 & -0.05 \\
\hline & $180^{\circ}$ & -0.05 & -0.04 & -0.04 & -0.04 & 0.13 \\
\hline
\end{tabular}

Figure 6. RS multivoxel patterns are correlated with the activation patterns. $\boldsymbol{a}$, Example of the patterns of activation (left) and the repetition index across voxels (right) for movement at $45^{\circ}$ (top) and $90^{\circ}$ (bottom). These examples are taken from the axial plane (Talairach coordinates: $z=50$ ) of one of the participants. Blue arrows denote correlations between the two measures for the same movement ( 0.59 and 0.61 , respectively for the participant in the example), whereas red arrows denote correlations between the two measures for different movements $(-0.26$ and -0.09 for this participant). $\boldsymbol{b}$, The resulting matrix of CCs when comparing the patterns of activation and the repetition index in all possible directions of movement. The main diagonal corresponds to $C(s$ computation of both measures, the nonrepeated trials were divided into two separate groups: one for computing the repetition index, the other for the activation index. Our results remained significant $\left(t_{(10)}=5.01, p<0.001\right)$.

We further tried to assess, on a voxel-by-voxel basis, whether the direction of movement similarly affected both the voxel's level of activation and its repetition index. Had the two measures been uncorrelated, one might expect that $20 \%$ of the voxels would show that 
a 10 clusters per voxel

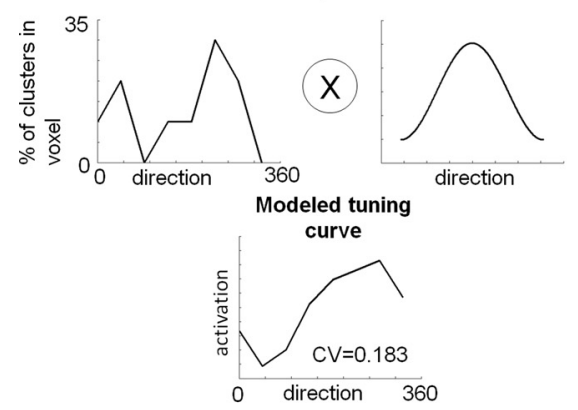

b

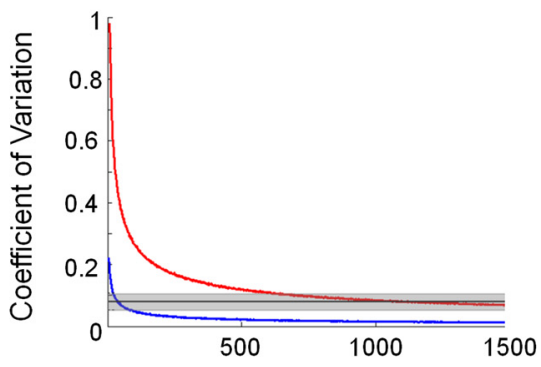

number of clusters per voxel
100 clusters per voxel

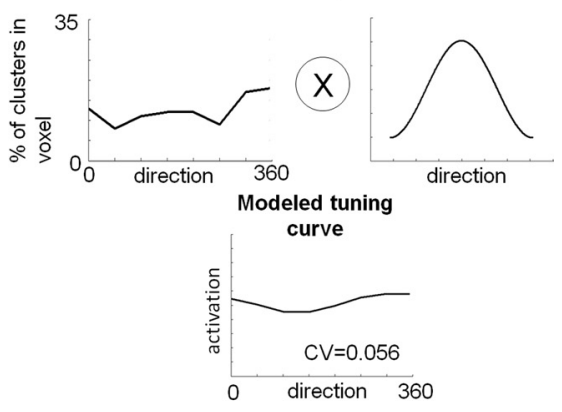

(Amirikian and Georgopoulos, 2003; Ben-Shaul et al., 2003; Stark et al., 2009).

\section{RS}

We find clear evidence for RS in human M1. This finding provides indirect evidence that the direction of movement is a key factor determining the response of neurons in the human motor cortex. A voxel's direction tuning is determined by two factors: the distribution of PDs across neuronal populations within the voxel and the average neuronal tuning width. Anisotropy of the distribution of PDs (due to the coarse-grained clustering of neuronal populations with similar PDs within a voxel) would result in direction preference at the voxel level. The most prevalent neuronal PD within the voxel's population of neurons will determine the voxel's PD. The voxel's tuning width, however, is affected both by the average neuronal tuning width and, possibly, by spatial correlations (i.e., greater tendency of a voxel to contain more neurons with PDs close to the most prevalent PD than neurons with far PDs).

In principle, RS may be helpful in distinguishing between the neuronal tuning characteristics of the voxel population and the spatial distribution of clusters, as the degree of RS is thought to be related to the average properties of single neurons (Grill-Spector and Malach, 2001). We find that the repetition effect is narrower than $45^{\circ}$; a decrease in activation is seen

the repetition index was maximal at the voxel's PD. We found that this was the case in $30 \%$ of the voxels, significantly more than that expected by chance $\left(t_{(10)}=4.52, p<0.0005\right)$.

\section{Discussion}

In this study, we show that M1 voxels are directionally tuned. This finding, at first, seems puzzling: based on an estimation of $\sim 50,000$ neurons in $1 \mathrm{~mm}^{3}$ of cortex (Beaulieu and Colonnier, 1983), there are on the order of 1,000,000 neurons in each voxel. If neuronal PDs are uniformly distributed across the population, one would expect that any fluctuations in firing rates would average out, and the overall activity during different movements would be the same. Thus, no directional preference should be seen at the voxel level.

The finding that voxels do show selectivity to direction of movement suggests that the spatial distribution of the neuronal PDs is not random. If neurons sharing the same PD are clustered in a columnar fashion, a voxel should contain many fewer independent elements. Under such circumstances, directional preference at the voxel level may not be that surprising. A similar preference of voxels in the human cortex has been seen in areas with known columnar organization in the monkey, such as V1 (for orientation) (Kamitani and Tong, 2005) and MT (for direction of motion) (Kamitani and Tong, 2006). Our results are also consistent with electrophysiological evidence in monkeys suggesting some degree of clustering of neurons in M1, based on their direction selectivity only for a movement with the same direction as in the previous trial, while a movement $45^{\circ}$ away from the previous one does not lead to any significant activation suppression. In this case, we would conclude that the tuning width of the voxel is determined mostly by spatial correlations. However, this finding is at odds with the broad tuning of neurons in macaque M1, which are well fit by a cosine waveform. It is possible that in human M1 the average neuronal tuning curves are narrower than in primates, as has been recently found for frequency tuning in auditory cortex (Bitterman et al., 2008). However, an alternative interpretation is that the relationship between RS and single-cell properties for actions is more complicated than the models so far proposed for sensory inputs (Grill-Spector et al., 2006). It is also possible that the RS could reflect the tuning properties of the inputs from other areas (Sawamura et al., 2006), and that the narrower width of the RS effect is a reflection of the narrower tuning curves in visual or parietal areas or their limited receptive field size (Heggelund and Albus, 1978; Andersen et al., 1985).

\section{Estimating cluster size}

One central goal in this paper was to roughly estimate the size of the average direction-selective cluster in M1 from our data. Consequently, we constructed a model with some simplifying assumptions. We assumed that M1 neurons have preference for one of eight discrete directions (which differ by $45^{\circ}$ ), and the 
direction tuning curve can be approximated by a cosine function [in which the mean baseline activity and modulation index were taken from Georgopoulos et al. (1982)]. These neuronal parameters were used to describe the tuning properties of an entire cluster, since a cluster was assumed to contain only neurons with the same PD. We also assumed that the PDs of the different clusters are uniformly distributed. However, as the number of clusters per voxel becomes smaller, random picks from this uniform distribution are likely to generate an uneven number of clusters tuned to each of the eight possible directions (Fig. 7a). This would lead to a preference for a specific direction at the voxel level, which would be smoothed to an extent by the characteristic tuning function of the neurons. One can therefore calculate the mean $\mathrm{CV}$ of each voxel as a function of the number of clusters within that voxel. The $\mathrm{CV}$ thus provides a measure of the modulation in the voxel's activation for the different directions, as depicted in Figure $7 a$ for the cases in which there were 10 or 100 clusters in a voxel.

Note that the average voxel's CV drops sharply as the number of direction-tuned clusters increases (Fig. $7 b$, blue and red curves). The voxel's CV, however, also depends on the average neuronal tuning width. If tuning is wide (e.g., cosine tuning, blue curve), the neurons also respond to other directions besides the $\mathrm{PD}$. This will obviously dampen variation in firing rates caused by a nonhomogeneous distribution of PDs. When the average neuronal tuning width was taken to follow a cosine fit, the value closest to the mean CV obtained in our experiment was $\sim 40$ clusters per voxel.

It is possible that neuronal tuning is in fact sharper than a cosine waveform (Amirikian and Georgopulos, 2000). To find the lower bound on cluster size, we recalculated the expected voxel $\mathrm{CV}$ as a function of the cluster number assuming an extremely narrow neuronal tuning $\left(<45^{\circ}\right)$ (Fig. $7 b$, red curve). In this case, $\sim 1200$ direction-selective clusters within a voxel are necessary to account for the variation observed in our fMRI data.

It should be taken into consideration that our model assumes no correlation between the tuning curves of neighboring clusters. Accounting for such correlations might lead to an estimation of a smaller cluster size. Notwithstanding these limitations, our estimates offer an upper and lower bound (at least in terms of orders of magnitude) on the size of an individual direction-selective cluster within human M1. If we further assume that the same PD is represented across all cortical layers (Hubel and Wiesel, 1962) and that the human cortex is $\sim 2.5 \mathrm{~mm}$ thick, one can ignore the third (depth) dimension of our $3 \times 3 \times 3 \mathrm{~mm}^{3}$ voxels. Thus, in the $3 \times 3 \mathrm{~mm}^{2}$ of voxel surface area, there are $\sim 40$ clusters per voxel, which translate to a cluster diameter of $\sim 470 \mu \mathrm{m}$. On the other hand, 1200 clusters per voxel (assuming a narrow tuning curve) would indicate that cluster diameter is $\sim 85 \mu \mathrm{m}$.

Interestingly, a similar estimation is found when using tuning curves of the average LFP elicited by movements in eight directions in monkey M1 (de Oliveira et al., 2001) (see Materials and Methods). The CV in this case was 0.2 , which corresponded to 7 or 185 clusters per voxel [assuming cosine or narrow $\left(<45^{\circ}\right)$ neuronal tuning width, respectively]. Assuming the LFP covers a surface area of $1 \mathrm{~mm}^{2}$ (Berens et al., 2008; Rasch et al., 2009), cluster diameter is estimated to be 75-380 $\mu \mathrm{m}$. Our estimation of the human M1 cluster diameter, in the range of $85-470 \mu \mathrm{m}$, nicely concurs with the order of magnitude of cluster size in the monkey estimated here (using the above LFP measures) as well as others (Amirikian and Georgopoulos, 2003; Stark et al., 2009).
Finally, it is important to remember that other components in this study, besides the direction of hand movement, could have influenced our results. These include the visual effects of target location, eye movements (which are likely to vary according to the target position), or visual aspects of the cursor movement. Therefore, from our current results we cannot unequivocally ascertain whether the neuronal selectivity is due to limb movement direction per se, or perhaps visual (or oculomotor) aspects of directionality. We shall refer to these important issues, in depth, elsewhere. It is also impossible to distinguish whether the reported activation is due mostly to neuronal activity during movement preparation ("Hold" period) or during the movement itself. This issue requires further investigation.

\section{Conclusions}

We show that the fMRI signal of individual voxels in human M1 is sensitive to the directional aspects of the reaching task and manifests direction-specific adaptation. Furthermore, the spatial patterns of the fMRI response are more correlated as movement directions are closer. We conclude that human M1 neurons are organized in clusters according to their PD. The model we constructed to estimate cluster size suggests that cluster diameter is likely to be of the order of a few hundred micrometers, which resembles the diameter estimated in monkeys.

\section{References}

Albright TD, Desimone R, Gross CG (1984) Columnar organization of directionally selective cells in visual area MT of the macaque. J Neurophysiol 51:16-31.

Amirikian B, Georgopoulos AP (2000) Directional tuning profiles of motor cortical cells. Neurosci Res 36:73-79.

Amirikian B, Georgopoulos AP (2003) Modular organization of directionally tuned cells in the motor cortex: is there a short-range order? Proc Natl Acad Sci U S A 100:12474-12479.

Andersen RA, Essick GK, Siegel RM (1985) Encoding of spatial location by posterior parietal neurons. Science 230:456-458.

Asanuma H, Rosén I (1972) Topographical organization of cortical efferent zones projecting to distal forelimb muscles in the monkey. Exp Brain Res 14:243-256.

Beaulieu C, Colonnier M (1983) The number of neurons in the different laminae of the binocular and monocular regions of area-17 in the cat. J Comp Neurol 217:337-344.

Ben-Shaul Y, Stark E, Asher I, Drori R, Nadasdy Z, Abeles M (2003) Dynamical organization of directional tuning in the primate premotor and primary motor cortex. J Neurophysiol 89:1136-1142.

Berens P, Keliris GA, Ecker AS, Logothetis NK, Tolias AS (2008) Feature selectivity of the gamma-band of the local field potential in primate primary visual cortex. Front Neurosci 2:199-207.

Berman NEJ, Wilkes ME, Payne BR (1987) Organization of orientation and direction selectivity in areas 17 and 18 of cat cerebral cortex. J Neurophysiol 58:676-699.

Bitterman Y, Mukamel R, Malach R, Fried I, Nelken I (2008) Ultra-fine frequency tuning revealed in single neurons of human auditory cortex. Nature 451:197-201.

Brainard DH (1997) The Psychophysics Toolbox. Spat Vis 10:433-436.

Cardoso de Oliveira S, Gribova A, Donchin O, Bergman H, Vaadia E (2001) Neural interactions between motor cortical hemispheres during bimanual and unimanual arm movements. Eur J Neurosci 14:1881-1896.

Dale AM (1999) Optimal experimental design for event-related fMRI. Hum Brain Mapp 8:109-114.

Dinstein I, Gardner JL, Jazayeri M, Heeger DJ (2008) Executed and observed movements have different distributed representations in human aIPS. J Neurosci 28:11231-11239.

Georgopoulos AP, Kalaska JF, Caminiti R, Massey JT (1982) On the relations between the direction of two-dimensional arm movements and cell discharge in primate motor cortex. J Neurosci 2:1527-1537.

Georgopoulos AP, Merchant H, Naselaris T, Amirikian B (2007) Mapping 
of the preferred direction in the motor cortex. Proc Natl Acad Sci U S A 104:11068-11072.

Grill-Spector K, Malach R (2001) fMR-adaptation: a tool for studying the functional properties of human cortical neurons. Acta Psychologica 107:293-321.

Grill-Spector K, Henson R, Martin A (2006) Repetition and the brain: neural models of stimulus-specific effects. Trends Cogn Sci 10:14-23.

Heggelund P, Albus K (1978) Response variability and orientation discrimination of single cells in striate cortex of cat. Exp Brain Res 32:197-211.

Hubel DH, Wiesel TN (1962) Receptive fields, binocular interaction and functional architecture in the cat's visual cortex. J Physiol 160:106-154.

Imig TJ, Adrian HO (1977) Binaural columns in primary field (A1) of cat auditory cortex. Brain Res 138:241-257.

Kamitani Y, Tong F (2005) Decoding the visual and subjective contents of the human brain. Nat Neurosci 8:679-685.

Kamitani Y, Tong F (2006) Decoding seen and attended motion directions from activity in the human visual cortex. Curr Biol 16:1096-1102.

Mehring C, Rickert J, Vaadia E, Cardoso de Oliveira S, Aertsen A, Rotter S (2003) Inference of hand movements from local field potentials in monkey motor cortex. Nat Neurosci 6:1253-1254.
Op de Beeck HP (2010) Against hyperacuity in brain reading: spatial smoothing does not hurt multivariate fMRI analyses? Neuroimage 49:1943-1948.

Pelli DG (1997) The Video Toolbox software for visual psychophysics: transforming numbers into movies. Spat Vis 10:437-442.

Powell TP, Mountcastle VB (1959) Some aspects of the functional organization of the cortex of the postcentral gyrus of the monkey: a correlation of findings obtained in a single unit analysis with cytoarchitecture. Bull Johns Hopkins Hosp 105:133-162.

Rasch M, Logothetis NK, Kreiman G (2009) From neurons to circuits: linear estimation of local field potentials. J Neurosci 29:13785-13796.

Rickert J, Cardoso de Oliveira S, Vaadia E, Aertsen A, Rotter S, Mehring C (2005) Encoding of movement direction in different frequency ranges of motor cortical local field potentials. J Neurosci 25:8815-8824.

Sawamura H, Orban GA, Vogels R (2006) Selectivity of neuronal adaptation does not match response selectivity: a single-cell study of the fMRI adaptation paradigm. Neuron 49:307-318.

Stark E, Drori R, Abeles M (2009) Motor cortical activity related to movement kinematics exhibits local spatial organization. Cortex 45:418-431.

Truccolo W, Friehs GM, Donoghue JP, Hochberg LR (2008) Primary motor cortex tuning to intended movement kinematics in humans with tetraplegia. J Neurosci 28:1163-1178. 